\title{
Diagnostic Performance of Serial bedside Capillary Lactate, Hemoglobin, and Shock Index for Severe Postpartum
}

\author{
Roberto Arturo Castillo-Reyther ${ }^{1}$, Idelia Natalie Plata-Alcocer ${ }^{1}$, Salvador De la Maza-Labastida ${ }^{1}$,

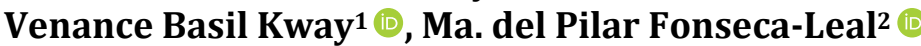 \\ ${ }^{1}$ Department of Obstetrics and Gynecology, Hospital Central Dr. Ignacio Morones Prieto, San Luis Potosi, Mexico \\ ${ }^{2}$ Research and Clinical Postgraduate Department of the Faculty of Medicine, Universidad Autonoma, de San Luis Potosi, Mexico \\ Email: venancedeverdad@alumnos.uaslp.edu.mx
}

How to cite this paper: Castillo-Reyther, R.A., Plata-Alcocer, I.N., De la MazaLabastida, S., Kway, V.B. and Fonseca-Leal, Ma.P. (2021) Diagnostic Performance of Serial bedside Capillary Lactate, Hemoglobin, and Shock Index for Severe Postpartum. Advances in Reproductive Sciences, 9 , 189-198.

https://doi.org/10.4236/arsci.2021.94018

Received: October 13, 2021

Accepted: November 27, 2021

Published: November 30, 2021

Copyright $\odot 2021$ by author(s) and Scientific Research Publishing Inc. This work is licensed under the Creative Commons Attribution International License (CC BY 4.0).

http://creativecommons.org/licenses/by/4.0/

\begin{abstract}
Objective: To assess the diagnostic capacity of bedside capillary lactate (CLact), capillary Haemoglobin ( $\mathrm{CHb})$, and shock index (SI) for severe postpartum haemorrhage (SPPH $>2000 \mathrm{ml}$ ) at diagnosis, 15 minutes and $30 \mathrm{mi}-$ nutes later. Method: A cohort study was carried out in a reference hospital in San Luis Potosi, Mexico from February 2020 to March 2021, and included sixty women in vaginal labor or c-section who presented PPH $(\geq 500 \mathrm{ml}$ in labor or $\geq 1000 \mathrm{ml}$ in c-section) measured by the gravimetric method. CLact, SI, and $\mathrm{CHb}$ concentrations were analyzed at diagnosis of PPH, 15 minutes, and 30 minutes. Patients who presented total blood loss of $>2000 \mathrm{ml}$ were considered SPPH. A T-test or Wilcox test was performed to compare the groups of non-severe and severe. Sensitivity, specificity, and performance were calculated by A Receiver Operating Curve. Results: A CLact measurement at 30 minutes was significantly different between the non-severe and severe groups $(4.0+1.9$ vs $4.8+1.15 \mathrm{P}$-value 0.001 , with an optimal cut point of $4.3 \mathrm{mmol} / \mathrm{dl}$ at AUC 0.75 , sensitivity 0.85 , and specificity of 0.62 . With a cut-point of 1.17, an AUC of 0.76 , sensitivity of 0.43 , and specificity of 0.98 , SI at diagnosis was significantly different between the non-severe and severe groups $(0.70+0.20$ vs. $0.90+0.38 \mathrm{P}$-value 0.0228$)$. Conclusion: $\mathrm{SI}$ is an early sign of SPPH; CLact can significantly identify SPPH after 30 minutes.
\end{abstract}

\section{Keywords}

Capillary, Haemoglobin, Lactate, Obstetric Haemorrhage, Shock Index

\section{Introduction}

Postpartum haemorrhage is the leading cause of maternal mortality and mor- 
bidity in the world accounting for approximately $10 \%$ of all births and the most common form of obstetric haemorrhage, which traditionally has been defined as a blood loss greater than $500 \mathrm{ml}$ after vaginal delivery or $1000 \mathrm{ml}$ after caesarean section [1]. In 2017 the American College of Gynecology and Obstetrics (ACOG) published a recent definition as a cumulative blood loss greater than or equal to $1000 \mathrm{ml}$ or a blood loss accompanied by signs or symptoms of hypovolemia within 24 hours of the delivery regardless of the route of birth [1]. Some institution still considers the traditional definition as appropriate [2]. In most developing countries, maternal mortality, and morbidity due to obstetric haemorrhage are caused by difficulties in early identification of the causes and lack of timely and adequate treatment. During the medical management of these cases, it is of great importance the early recognition of patients with severe cases o may develop severe haemorrhage [3] [4]. For this reason, Obstetricians need to have reliable bedside clinical and biochemical tools to identify patients at risk of a critical condition if they are not provided immediate resuscitation. The schemes studied for this purpose include non-capillary schemes such as the shock index, venous serum lactate and Haemoglobin levels. Some studies reported the combination of these schemes to be useful but not studied at the capillary level for bedside use [5]. It is well established that SI correlates with SPPH and is a good predictor for transfusion and complications in patients with hypovolemic shock. [5] [6] [7] [8] [9] but it is also known that the vital signs are dynamic states, which means they can rapidly change over a short time, and the physiological changes during pregnancy may make vital signs not reliable for the early identification of severe haemorrhage as they are lately modified [10], the calculation of SI involves the use of vital signs (heart rate divided by systolic blood pressure), for this reason, we investigate whether the SI is consistently useful over time as an indicator for SPPH.

On other hand, venous serum lactate has been used as a biochemical marker to guide fluid recusation in patients with postpartum haemorrhage and it has also been established as an indicator of severe postpartum haemorrhage [5] [11]. The clinical use of portable devices to measure capillary lactate seems to be an easy and quick way to detect lactate compared to conventional venous laboratory testing. Acuttrend Lactate $^{\circledR}(\mathrm{AL})$ is a portable device for measuring capillary lactate, its reliability was compared versus the EBIO plus analyzer as a gold standard showed to have good reliability for intra, inter-analyzers, and between test strips (ICC $r=0.999$ ) at different lactate concentrations; coefficient of variation reported between $1.8 \%$ and 3.3\% [12] Capillary hemodynamics may vary in time for its establishment compared to venous dynamics as it involves microcirculation. For this reason, it is genuine to investigate whether the capillary lactate can also be an early predictor of SPPH within the first 30 minutes from the diagnosis of PPH. Haemoglobin concentration is one of the criteria for transfusion.

Recently, the use of optical noninvasive real-time capillary Haemoglobin 
monitoring is becoming popular in surgery and suggested to be used as a guide for transfusions [13], It is reported that women without $\mathrm{PPH}$, the mean $\mathrm{Hb}$ decrease is approximately $1 \mathrm{~g} / \mathrm{dl}$, while a $2 \mathrm{~g} / \mathrm{dl}$ to $3 \mathrm{~g} / \mathrm{dl}$ decreases are commonly observed in women with occult PPH or overt PPH, respectively [14]. But this drop of Haemoglobin is neither described in terms of time nor in capillary level. Early drop of capillary Haemoglobin is not yet established as a predictor of $\mathrm{SPHH}$ in terms of its change with time considering the phenomenon of the redistribution for this reason we aim at investigating the cut point of bedside capillary Haemoglobin drop as an indicator for severe haemorrhage at the diagnosis, 15 min and within first 30 minutes time intervals after diagnosis. HemoCue is a portable device which provides reliable measurements, especially within the critical $6-10 \mathrm{~g} / \mathrm{dL}$ range, with red compared with standard laboratory test mean difference of $0.54(0.52-0.81)$ [15].

With the aim of getting the clinical bedside tools, we investigated the use of bedside and minimally invasive protocols by measuring capillary lactate and Haemoglobin with Acuttrend ${ }^{\circledR}$ Lactate and HemoCue ${ }^{\circledR}$ respectively. Accuracy and Reliability of the devices Acuttrend ${ }^{\circledR}$ Lactate and HemoCue ${ }^{\circledR}$, were previously reported in different studies. As a point of care that allows a quick dynamic evaluation accompanied by a clinical marker, we also integrated the shock index (SI).

\section{Method}

A prospective cohort study was carried out from February 2020 to March 2021 in which were included pregnant women with postpartum haemorrhage ( $\geq 500$ $\mathrm{ml}$ in labor or $\geq 1000 \mathrm{ml}$ in c-section, Institutional definition of postpartum haemorrhage) in a reference hospital in San Luis Potosi Mexico (Hospital Central Dr Ignacio Morones Prieto). The study obtained approval from the Institutional Ethical Committee (Registration number CONBIOETICA 93-19).

We included Pregnant patients in labor or cesarean section with postpartum haemorrhage, without conditions that might elevate lactate concentration such as eclampsia, leukemia, lymphoma, and solid tumors, poorly controlled diabetes, liver failure, and ingestion of antiretroviral drugs and biguanides, confirmed sepsis or diagnosed with severe shock or documented anemia at the beginning of the study, Patients who decided to withdraw the consent or to leave the study were eliminated. Bleeding volume was calculated by the gravimetric method as described here, blood-Stained Surgical Gauzes from delivery or c-section were weighed, and its dry weight was subtracted from the total blood-stained weight. Blood weight ( $\mathrm{g}$ ) was converted into blood volume $(\mathrm{ml})$ using the accepted approximation of blood density of $1 \mathrm{~g}=1 \mathrm{~mL}$. At the diagnosis of postpartum haemorrhage ( $\geq 500 \mathrm{ml}$ in labor or $\geq 1000 \mathrm{ml}$ in c-section, Institutional definition of postpartum haemorrhage), Capillary lactate and Haemoglobin were determined by bedside equipment Acuttrend plus $\odot$, and Shock index was calculated as the heart rate (HR) divided by systolic blood pressure (SBP). These parame- 
ters were also measured at 15 and 30 minutes after the diagnosis. At the end of the obstetric event the patients with total blood loss of $>2000$ were considered SPPH.

\section{Statistical Analysis}

Statistical analysis was performed using Statistical program R 3.6 version and R Studio 1.4.1717, version. The sample size was calculated by $\mathrm{r}$ package ("MKmisc"), with command power, diagnostic, test, for diagnostic test with sensitivity of 0.95 , specificity of 95 Significant level $=0.05$, delta $=0.1$, power $=0.8$ as suggested by Cohen. The sample size required was 68 patients. We considered appropriate including with 60 patients as it does not alter the statistical power. The distribution of continuous data was assessed by Shapiro-Wilk's test, a comparison of capillary Haemoglobin, lactate, and shock index values between severe and non-severe haemorrhage groups was done by t-Test in case of normal distribution and Wilcox test for non-normal distribution data. A Receiver Operating Curve analysis was done to determine cut points by Youden's index method, Sensitivity, specificity, and performance of each test at 0,15 , and $30 \mathrm{mi}$ nutes from the diagnosis.

\section{Results}

We included 60 patients, 27 (45\%) cesarean section y 33 (55\%) vaginal delivery. Of all Patients, $18(30 \%)$ were classified as severe and $42(70 \%)$ as non-severe. (See Patients flow diagram). General mean blood loss was $1194.92+627.67 \mathrm{ml}$. Non severe $983.52+354.64 \mathrm{ml}$ and severe group $2392.77+469.48 \mathrm{ml}$. The most common cause of SPPH was uterine atony 29 (48.05\%), Other general characteristics of the study groups are shown in Table 1 . SI at the diagnosis was significantly differentiate between severe from non-severe group $(0.70+0.20$ vs. $0.90+$ $0.38 \mathrm{P}$-value 0.0228 ) (Table 2). Its cut point of 1.17 has shown good performance with AUC of 0.76 , sensitivity of 0.43 and specificity of 0.98 (Figure 2). The same as SI at 30 minutes $(0.70+0.27$ vs. $0.90+0.55 \mathrm{P} 0.0228)$ (Table $2 \&$ Figure 1). Capillary lactate only at $30 \mathrm{~min}$ was able to differentiate non-severe from severe group $\left(4.0+1.90\right.$ vs. $\left.4.8+1.15 \mathrm{p}<0.001^{\star}\right)$. Its cut points of 4.30 $\mathrm{mmol} / \mathrm{dl}$ at $30 \mathrm{~min}$ showed good performance (AUC 0.75 ), with sensitivity 0.85 , specificity 0.62 . Whereas at the diagnosis offers unsatisfactory performance (AUC 0.46 ) $5.50 \mathrm{mmol} / \mathrm{dl}$, Specificity 0.62 , Sensitivity 0.8 and at $15 \mathrm{~min}$ also had the same performance $(0.46) 5.5 \mathrm{mmol} / \mathrm{dl}$, AUC 0.46 , Sensitivity 0.73 specificity 0.43 (Table 2 \& Figure 2).

Capillary Haemoglobin was not able to differentiate group of severe from non-severe group as early as 30 minutes. Generally had unsatisfactory performance and showed unstable changes (positive and negative values see graph 1). Cut points of but $9.60 \mathrm{mg} / \mathrm{dl}$ at $30 \mathrm{~min}$ had satisfactory (AUC 0.62) with a specificity of 0.71 and sensitivity of 0.58 , although $8.40 \mathrm{mg} / \mathrm{dl}$ at $15 \mathrm{~min}$ was more sensitive (Sensitivity 0.90 , specificity 0.42 ) had unsatisfactory performance (AUC 
0.56), the same performance was obtained with 9.4 at the diagnosis (AUC 0.58), sensitivity 0.69 specificity 0.57 (Table $1 \&$ Figure 3, Figure 4 ).

Table 1. General characteristics of the study groups.

\begin{tabular}{|c|c|c|c|c|}
\hline VARIABLE & TOTAL & NON-SEVERE PPH & SEVERE PPH & P VALUE \\
\hline & $\mathrm{N}=60$ & $\mathrm{n}=42$ & $\mathrm{n}=19$ & \\
\hline & Mean \pm SD & Mean \pm SD & Mean \pm SD & \\
\hline & Median \pm IQR & Median \pm IQR & Median \pm IQR & \\
\hline Gestational Age (Weeks) & $38.70 \pm 2.10$ & $38.5 \pm 2.84$ & $38.7 \pm 1.97$ & $0.8441 \mathrm{t}$ \\
\hline Crystalloids (ml) & $2061 \pm 781$ & $2537.14 \pm 795.50$ & $1999.21 \pm 513.00$ & $0.0355^{\star} \mathrm{W}$ \\
\hline Maternal Age (years) & $25.0 \pm 6.00$ & $32.0 \pm 7.90$ & $24 \pm 5.70$ & $0.4261 \mathrm{t}$ \\
\hline Cesarean & $27(45.03 \%)$ & $18(43.67 \%)$ & $9(47.02 \%)$ & $0.5804 x$ \\
\hline Hypertensive desorders & $8(13.33 \%)$ & $6(14.32 \%)$ & $2(10.33 \%)$ & $0.8909 \mathrm{x}$ \\
\hline Gestacional Diabetes & $11(18.45 \%)$ & $3(7.08 \%)$ & $8(42.89 \%)$ & $0.3670 \mathrm{x}$ \\
\hline Multiple gestation & $3(5.06 \%)$ & $1(2.67 \%)$ & $2(10.09 \%)$ & $0.2001 \mathrm{x}$ \\
\hline Total Blood loss (ml) & $1194.92 \pm 627.67$ & $983.52 \pm 354.64$ & $2392.77 \pm 469.48$ & $<0.001{ }^{\star} \mathrm{t}$ \\
\hline \multicolumn{5}{|l|}{ Causes of PPH } \\
\hline Atony & $29(48.05 \%)$ & $17(40.34 \%)$ & $9(49.03 \%)$ & \\
\hline Retention & $13(22.04 \%)$ & $5(11.54 \%)$ & $8(42.23 \%)$ & $0.8918 \mathrm{x}$ \\
\hline Trauma & $18(30.34 \%)$ & $7(15.43 \%)$ & $11(58.43 \%)$ & \\
\hline
\end{tabular}

w-wilcox test for non-normal data, $\mathrm{t}-\mathrm{T}$ test for normal data, IC: Shock index. X: Chisq test. Lact: Capillary lactate. Hb: Hemoglobin Dx: Diagnosis. ${ }^{*}$-statistically significant.

Table 2. Behavior of capilary lactate, shock index and capilarry hemoglobin.

\begin{tabular}{|c|c|c|c|c|}
\hline VARIABLE & $\begin{array}{c}\text { TOTAL } \\
\text { MEAN } \pm \text { SD } \\
\text { MEDIA } \pm \text { IQR }\end{array}$ & $\begin{array}{c}\text { NON-SEVERE PPH } \\
\text { MEAN } \pm \text { SD } \\
\text { MEDIA } \pm \text { IQR }\end{array}$ & $\begin{array}{c}\text { SEVERE PPH } \\
\text { MEAN } \pm \text { SD } \\
\text { MEDIA } \pm \text { IQR }\end{array}$ & P VALUE \\
\hline $\mathrm{S} 1$ at $\mathrm{Dx}$ & $0.70 \pm 0.30$ & $0.70 \pm 0.20$ & $0.90 \pm 0.38$ & $0.0228^{\star} \mathrm{w}$ \\
\hline $\mathrm{SI}$ at $15 \mathrm{~min}$ & $0.70 \pm 0.22$ & $0.70 \pm 0.20$ & $0.80 \pm 0.23$ & $0.1717 \mathrm{w}$ \\
\hline SI at $30 \mathrm{~min}$ & $0.70 \pm 0.20$ & $0.70 \pm 0.27$ & $0.90 \pm 0.55$ & $0.0228^{\star} \mathrm{w}$ \\
\hline Lactate $\mathrm{Dx}(\mathrm{mmol} / \mathrm{dl})$ & $4.80 \pm 2.68$ & $4.8 \pm 2.60$ & $5.6 \pm 3.30$ & $0.7645 \mathrm{w}$ \\
\hline Lactate $15 \mathrm{~min}(\mathrm{mmol} / \mathrm{dl})$ & $4.40 \pm 2.58$ & $4.4 \pm 2.80$ & $4.5 \pm 2.40$ & $0.9174 w$ \\
\hline Lactate $30 \mathrm{~min}(\mathrm{mmol} / \mathrm{dl})$ & $4.10 \pm 2.58$ & $4.0 \pm 1.90$ & $4.8 \pm 1.15$ & $<0.001^{\star} \mathrm{w}$ \\
\hline $\mathrm{Hb}$ at $\mathrm{Dx}(\mathrm{mg} / \mathrm{dl})$ & $10.38 \pm 2.13$ & $10.43 \pm 2.16$ & $9.98 \pm 1.84$ & $0.5738 \mathrm{t}$ \\
\hline $\mathrm{Hb} 15 \mathrm{~min}(\mathrm{mg} / \mathrm{dl})$ & $10.37 \pm 1.74$ & $10.43 \pm 2.30$ & $9.91 \pm 4.35$ & $0.5897 \mathrm{t}$ \\
\hline $\mathrm{Hb} 30$ (mg/dl) & $9.74 \pm 1.70$ & $9.83 \pm 1.70$ & $9.10 \pm 1.57$ & $0.2915 \mathrm{t}$ \\
\hline Hb Change $15 \mathrm{~min}(\mathrm{mg} / \mathrm{dl})$ & $0.45 \pm 1.65$ & $0.3 \pm 1.92$ & $0.07 \pm 2.21$ & $0.4267 \mathrm{w}$ \\
\hline $\mathrm{Hb}$ change $30 \mathrm{~min}(\mathrm{mg} / \mathrm{dl})$ & $0.55 \pm 2.25$ & $1.3 \pm 1.85$ & $0.5 \pm 2.25$ & $0.6204 \mathrm{w}$ \\
\hline
\end{tabular}

w-wilcox test for non-normal data, $\mathrm{t}-\mathrm{T}$ test for normal data, IC: Shock index. Lact: Capillary lactate. Hb: Hemoglobin Dx: Diagnosis. ${ }^{*}$ - statistically significant. 


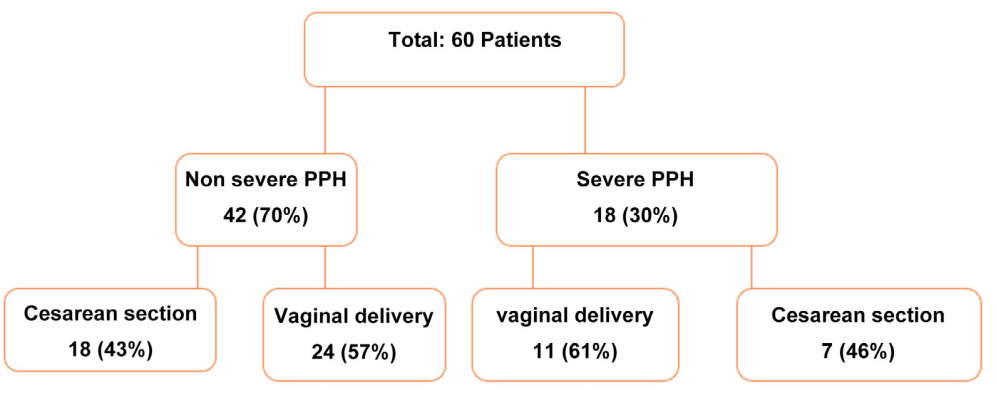

Figure 1. Patientes flow diagram.

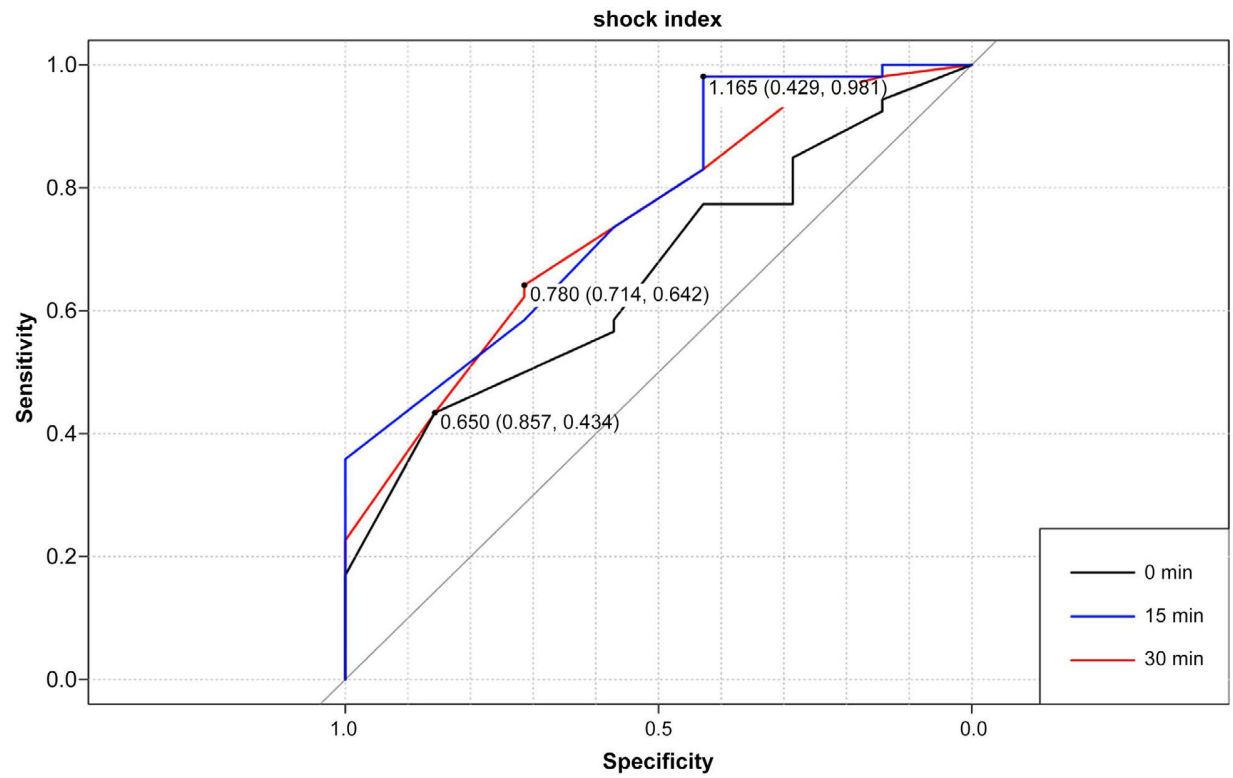

Figure 2. Receiver operating curve: cut points for capillary lactate at 0-, 15- and 30-minutes post diagnosis of $\mathrm{PPH}$.

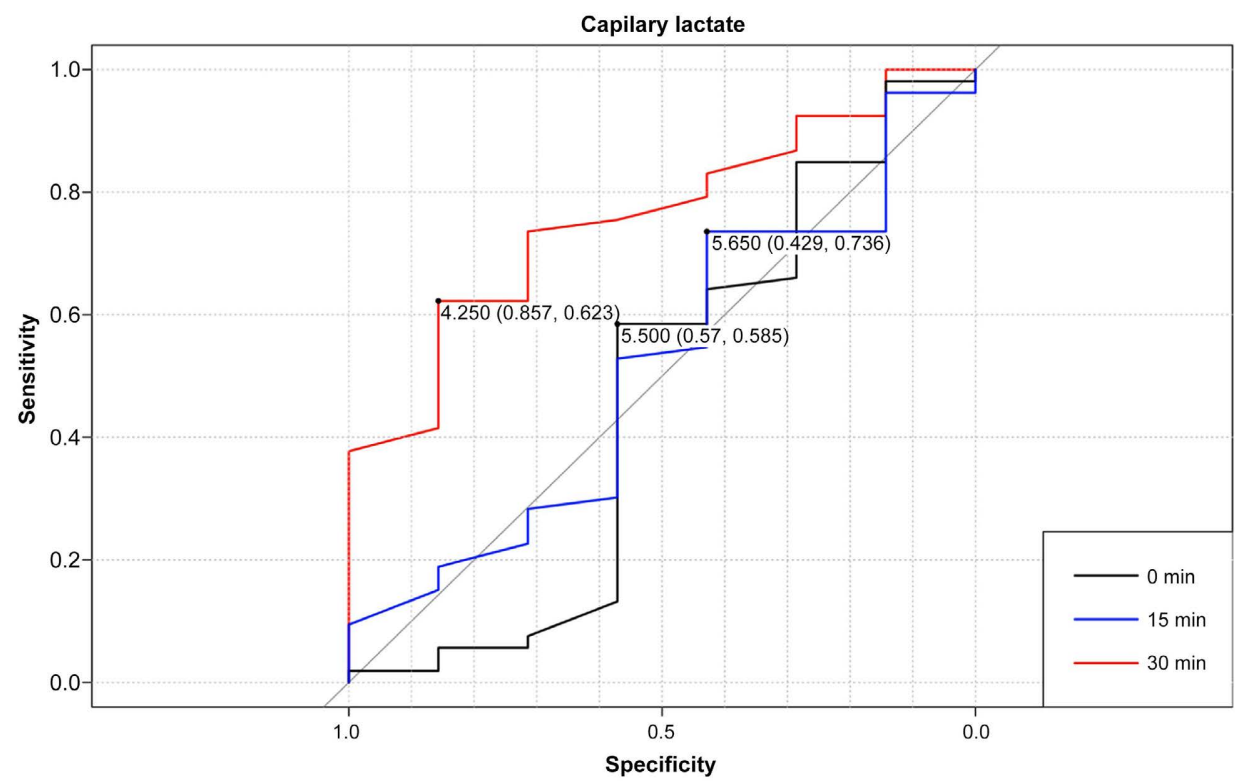

Figure 3. Receiver operating curve: cut points for SI at 0-, 15- and 30-minutes post diagnosis of $\mathrm{PPH}$. 


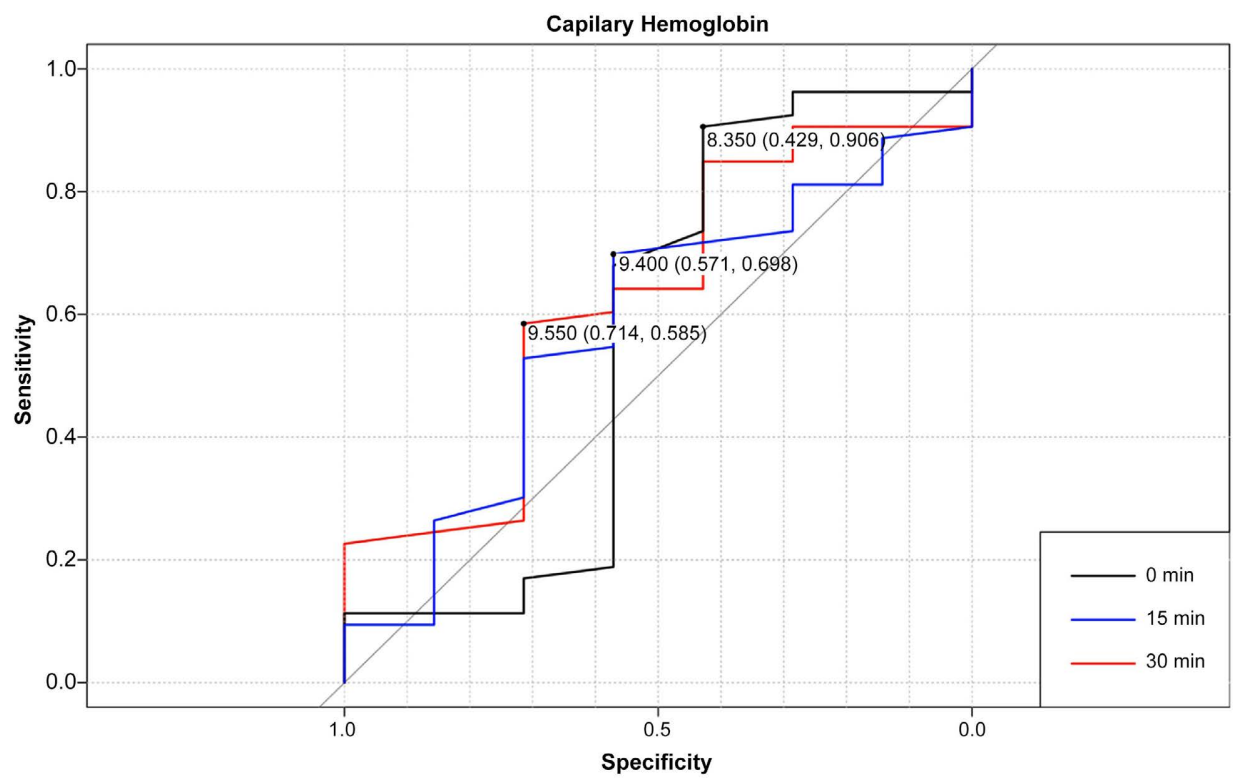

Figure 4. Receiver operating curve: cut points for capillary hemoglobin at 0-, 15- and 30minutes post-diagnosis of $\mathrm{PPH}$.

\section{Discussion}

Results of this study have shown the capacity of shock index, capillary lactate in identifying severe postpartum haemorrhage defined as blood loss of more than $2000 \mathrm{ml}$. Additionally, reports an optimal moment for analysis of these parameters. Shock index is defined as the heart rate divided by systolic blood pressure, has been studied in patients e at risk of or experiencing shock from a variety of causes such as trauma, myocardial infarction, haemorrhage, pulmonary embolism, sepsis, and obstetric haemorrhage. Schroll R. Et al reported Shock Index $\geq 1$ had a sensitivity of $67.7 \%$ (95\% CI $49.5 \%-82.6 \%)$ and specificity of $81.3 \%$ (95\% CI $78.0 \%-84.3 \%$ ) for predicting massive transfusion in trauma patients. In obstetrics, Nathan et al reported that SI $\geq 1.7$ had $25.0 \%$ sensitivity (95\% CI 5.5 57.2 ) and $97.7 \%$ specificity (CI 94.8 - 99.3), for predicting ICU admission in postpartum haemorrhage [13]. Welsh et al reported SI de 0.9 as a good predictor of necessity for transfusion in postpartum haemorrhage [14]. Most of these studies which investigated the clinical application of SI have not determined an optimal moment for its determination. This study determined that the value of the shock index, dynamically evaluated, at the time of diagnosis, and 30 minutes after diagnosis, allows early identification of those patients who present massive bleeding with the sensitivity of 0.43 and specificity of 0.98 and 0.64 sensitivity and specificity 0.7 respectively. Our study's cut point did not differ much from other studies. Generally, we recommend considering SI $>1$ as the predictor of adverse effects in most of the clinical scenarios independent of the moment of its determination. At initial phases of shock, the compensatory mechanism of the cardiovascular system responds by increasing the heart rate, increasing myocardial contractility, and constricting peripheral blood vessels as a result of the direct stimulation via the sympathetic system this compensatory mechanism 
maintains the stable blood pressure and elevated heart rate, these mechanisms make the shock index an early indicator of severe haemorrhage. Serum lactate has been studied as a strong biochemical marker of tissue hypoperfusion in obstetric haemorrhage; increased lactate concentration may indicate hypovolemic shock before traditional markers such as hemoglobin concentrations, and vital signs in pregnant women. Previously it was reported that venous lactate of 2.6 $\mathrm{mmol} / \mathrm{dl}$ at the diagnosis of postpartum haemorrhage may be a good predictor for severe haemorrhage (sensitivity of 0.85 and specificity was 0.76 ) [10]. We found that at the capillary level the cut point of $4.3 \mathrm{mmol} / \mathrm{dl}$ at $30 \mathrm{~min}$ had good performance (AUC 0.75 ), with sensitivity 0.85 , specificity 0.62 AUC 0.75 . At the diagnosis and 15 min we obtained higher cut points but with low performance. High cut points for capillary lactate compared to venous lactate may be due to multifactorial effects including lactate detection methods and compensatory mechanisms of peripheric blood constriction to guarantee blood flow to vital organs. There are portable lactate analyzers that require $15-50 \mu \mathrm{L}$ of blood and take approximately 60 seconds to process which makes it easier for the bedside monitoring by capillary lactate. Putting into practice our results, the bedside serum capillary lactate as a point-of-care test can differentiate the severe group from non-severe to allow quick during lactate-guided recusation and probably reduce risk of thrombophlebitis that may result from venous sampling capillary hemoglobin was not statistically significant in differentiating between patients with severe bleeding. Had unstable changes (i.e., with negative and positive values) may explain that the capillary hemodynamics y phenomena de redistributions of hemoglobin affect the hemoglobin concentration at this level. we found that a capillary hemoglobin value of 8.4 at 15 minutes has a sensitivity of $90 \%$ and specificity of $42 \%$ for identification of patients who are at risk of reaching a critical condition if immediate resuscitation is not provided, and thus assist in the rapid and objective recognition of patients with severe postpartum haemorrhage (those who require massive transfusion during the immediate phase of resuscitation, including fluids and blood products) since patients with haemorrhage obstetrics requiring blood transfusion are a significant cause of maternal morbidity in our setting.

The limitation of this study is that it was carried out in a tertiary level hospital where there is the availability of human resources and materials to combat severe haemorrhage for this reason very few patients may present complications such as coagulopathy and needs for intensive care unit admission, for this reason, we cannot tell the exact risk those complications using our capillary scheme. On another side, one of its advantages is that the materials needed to obtain these results are portable devices that can be easily obtained in the market, and thus low resourced facilities can obtain them for their patient monitoring. Changes in capillary hemoglobin have shown to be inconsistent since had both positive and negative values which indicate instability of capillary dynamics that confirms the real nature of the hemoglobin redistribution.

Shock index and capillary Lactate may be reliable bedside clinical tools to 
identify patients with severe haemorrhage. Shock index is an early clinical detector of severe postpartum haemorrhage as early as at diagnosis, whereas lactate gains its value at 30 minutes post-diagnosis. Capillary hemoglobin was not able to differentiate the severe from non-severe as early as the first $30 \mathrm{~min}$.

\section{Conclusion}

Although the vital signs changes delay in identifying severe cases, SI is still an early sign of SPPH; CLact can significantly identify SPPH after 30 minutes probably due to different capillary hemodynamics compared to venous. Finally, capillary hemoglobin is not an early detector of SPPH probably due to the mechanism of hemoglobin redistribution. These three bedside markers may be combinedly used to optimize the diagnosis and management of SPPH. More studies are required in different populations to increase the external validity of these results.

\section{Acknowledgements}

We appreciate the cooperation of Resident Physicians of Obstetrics Gynecology from the Hospital Central "Dr. Ignacio Morones Prieto". San Luis Potosi. Mexico.

\section{Disclosures}

\section{Human Rights Statements and Informed Consent}

Helsinki dilaceration guidelines were adhered. All participants gave their consent by signing the informed consent.

\section{Approval by Ethics Committee}

The study obtained approval from the Institutional Ethical Committee (Registration number CONBIOETICA 93-19).

\section{Conflicts of Interest}

The authors declare no conflicts of interest regarding the publication of this paper.

\section{References}

[1] Committee on Practice Bulletins-Obstetrics (2017) Practice Bulletin No. 183: Postpartum Hemorrhage. Obstetrics \& Gynecology, 130, e168-e186. https://doi.org/10.1097/AOG.0000000000002351

[2] Sentilhes, L., Merlot, B., Madar, H., Sztark, F., Brun, S. and Deneux-Tharaux, C. (2016) Postpartum Haemorrhage: Prevention and Treatment. Expert Review of Hematology, 9, 1043-1061. https://doi.org/10.1080/17474086.2016.1245135

[3] Higgins, N., Patel, S.K. and Toledo, P. (2019) Postpartum Hemorrhage Revisited: New Challenges and Solutions. Current Opinion in Anaesthesiology, 32, 278-284. https://doi.org/10.1097/ACO.0000000000000717

[4] Lockhart, E. (2015) Postpartum Hemorrhage: A Continuing Challenge. Hematology-American Society of Hematology Education Program, 2015, 132-157. https://doi.org/10.1182/asheducation-2015.1.132 
[5] Lee, E.S., Kim, S.R., Seo, D.W., Won, H.-S., Shim, J.-Y., Lim, K.-S., et al. (2017) Serum Lactate and Shock Index in Primary Postpartum Hemorrhage: Serum Lactate Levels Combined with Shock Index Improve the Predictive Performance for Massive Transfusion in Patients with Primary Postpartum Hemorrhage. Journal of Emergency Medicine, 53, 439 p. https://doi.org/10.1016/j.jemermed.2017.08.053

[6] Lee, S., Kim, H., Cho, G., Hong, S., Oh, M. and Kim, H. (2018) Use of the Shock Index to Predict Maternal Outcomes in Women Referred for Postpartum Hemorrhage. International Journal of Gynecology \& Obstetrics, 144, 221-224. https://doi.org/10.1002/ijgo.12714

[7] Rau, C.-S., Wu, S.-C., Kuo, S., Kuo, P.-J., Hsu, S.-Y., Chen, Y.-C., et al. (2016) Prediction of Massive Transfusion in Trauma Patients with Shock Index, Modified Shock Index, and Age Shock Index. International Journal of Environmental Research and Public Health, 13, Article No. 683.

https://doi.org/10.3390/ijerph13070683

[8] Nathan, H., El Ayadi, A., Hezelgrave, N., Seed, P., Butrick, E., Miller, S., et al. (2015) Shock Index: An Effective Predictor of Outcome in Postpartum Haemorrhage? BJOG: An International Journal of Obstetrics \& Gynaecology, 122, 268-275. https://doi.org/10.1111/1471-0528.13206

[9] Welsh, A., Savala, M., Lai, B., Kimata, C. and Soon, R. (2018) Postpartum Shock Index as a Predictor of Postpartum Hemorrhage Morbidity [30K]. Obstetrics \& Gynecology, 131, 127S-127S. https://doi.org/10.1097/01.AOG.0000533532.21165.73

[10] Pacagnella, R.C. and Borovac-Pinheiro, A. (2019) Assessing and Managing Hypovolemic Shock in Puerperal Women. Best Practice \& Research Clinical Obstetrics \& Gynaecology, 61, 89-105. https://doi.org/10.1016/j.bpobgyn.2019.05.012

[11] Basil-Kway, V., Castillo-Reyther, R., Domínguez-Salgado, L.A., Espinosa-Tanguma, R., Medina, Ú. and Gordillo-Moscoso, A. (2020) Early Prognostic Capacity of Serum Lactate for Severe Postpartum Hemorrhage. International Journal of Gynecology \& Obstetrics, 153, 483-488. https://doi.org/10.1002/ijgo.13446

[12] Scafoglieri, A., Tresignie, J., Provyn, S., Clarys, J.P. and Bautmans, I. (2012) Reproducibility, Accuracy and Concordance of Accutrend ${ }^{\circledR}$ Plus for Measuring Circulating Lipid Concentration in Adults. Biochemia Medica, 22, 100-108. https://doi.org/10.11613/BM.2012.011

[13] Barker, S.J., Shander, A. and Ramsay, M.A. (2016) Continuous Noninvasive Hemoglobin Monitoring: A Measured Response to a Critical Review. Anesthesia \& Analgesia, 122, 565-572. https://doi.org/10.1213/ANE.0000000000000605

[14] Yefet, E., Yossef, A., Suleiman, A., Hatokay, A. and Nachum, Z. (2020) Hemoglobin Drop Following Postpartum Hemorrhage. Scientific Reports, 10, Article No. 21546.

[15] Johnson, M., Marwick, P.C. and Coetzee, J.F. (2020) Comparison of Hemoglobin Measurements by 3 Point-of-Care Devices With Standard Laboratory Values and Reliability Regarding Decisions for Blood Transfusion. Anesthesia \& Analgesia, 131, 640-649. https://doi.org/10.1038/s41598-020-77799-0 